\title{
Influencia de la aplicación de la norma G050 en el clima de seguridad de las obras de construcción del distrito de Surquillo
}

\author{
Influence of the application of the G050 norm in the safety climate of the \\ construction works of the Surquillo district
}

\author{
Edson F. Del Águila Guerrero ${ }^{1}$ \\ Recibido: Enero 2020 - Aprobado: Junio 2020 - Publicado: Junio 2020
}

\begin{abstract}
RESUMEN
La presente investigación tiene como objeto estudiar la relación entre la aplicación de la norma G050 (variable independiente) y el clima de seguridad de las obras de construcción del distrito de Surquillo (variable dependiente). La metodología utilizada corresponde al tipo de investigación aplicada, nivel evaluativo, con diseño no experimental (ex post-facto). La población de estudio son los trabajadores de 25 obras de construcción multifamiliares del distrito de Surquillo, y la muestra fue de 196 trabajadores escogidos mediante un muestreo aleatorio simple. La investigación demostró que la aplicación de la norma G050 influye en el Clima de Seguridad de las Obras de Construcción del Distrito de Surquillo por cuanto los promedios generales de la variable independiente y dependiente superan el valor central esperado, representando por tanto valores positivos; asimismo, a partir de la prueba de hipótesis se demostró una relación de dependencia de la variable V2 respecto a variable V1; por lo que las empresas del sector construcción deberán considerar estas variables en su gestión integral de SST con la finalidad de asegurar una efectiva prevención de pérdidas.
\end{abstract}

Palabras claves: Clima; disuasión; estructuras; identidad; legitimidad; norma; políticas; y seguridad.

\begin{abstract}
The purpose of this research is to study the relationship between the application of the G050 standard (independent variable) and the safety climate of construction works in the Surquillo district (dependent variable). The methodology used corresponds to the type of applied research, evaluative level, with a non-experimental design (ex post-facto). The study population is the workers of 25 multifamily construction works in the Surquillo district, and the sample was 196 workers chosen through simple random sampling. The investigation showed that the application of the G050 standard influences the Safety Climate of the Construction Works of the Surquillo District in that the general averages of the independent and dependent variable exceed the expected central value, therefore representing positive values; also, from the hypothesis test a dependency relation of the variable V2 with respect to variable V1 was demonstrated; therefore, companies in the construction sector should consider these variables in their comprehensive management of $\mathrm{OSH}$ in order to ensure effective loss prevention.
\end{abstract}

Keywords: Climate; deterrence; structures; identity; legitimacy; norm; policies; and security.

\footnotetext{
${ }^{1}$ Superintendencia Nacional de Fiscalización Laboral, Av. Arenales nº 815 - 821 Santa Beatriz, distrito Lima Cercado, Lima, Perú.

E-mail: Edson.delaguila@sunafil.gob.pe
} 


\section{INTRODUCCIÓN}

En los últimos años a partir de la creación de la Sunafil y la ejecución de su acción orientadora y fiscalizadora, la seguridad y salud en el trabajo en el sector construcción viene progresando positivamente; sin embargo, este desarrollo no ha sido suficiente, pues persiste una alta tasa de accidentes de trabajo en el sector construcción, tal como lo publica el "Boletín Estadístico Mensual de Notificaciones de Accidentes de Trabajo, Incidentes Peligrosos y Enfermedades Ocupacionales del Ministerio de Trabajo y Promoción del Empleo de Perú (MTPE)", el cual registra a agosto de 2019: trescientos veintinueve (329) notificaciones en el Sistema de Accidentes de Trabajo del MTPE, observándose dos (02) accidentes mortales, trescientos veintitrés (323) accidentes de trabajo, y cuatro (04) incidentes peligrosos (MTPE, s. f.).

En Surquillo uno de los distritos ubicados en la ciudad de Lima, durante el año 2016 se han registrado ciento sesenta y un (161) construcciones (Municipalidad de Surquillo, s. f.), hecho que demuestra un rápido crecimiento de esta actividad, y el consecuente incremento a la exposición a tareas de alto riesgo, propias de la construcción, al personal profesional y obrero que labora en dichas obras; asimismo, dicho distrito en los años 2014 y 2018 registró tres (03) accidentes mortales (El Comercio Perú, 2018) (América Noticias, s. f.).

La problemática expuesta tiene como origen la informalidad, y para nuestro particular caso, el inexistente estudio de la relación de la norma G050 Seguridad durante la construcción con el clima de seguridad; muy a pesar que (Bird, F. y Germain, G., s. f.) tras un estudio demuestra que el $85 \%$ de los accidentes de trabajo se deben a actos inseguros o fallas de las personas, y que (Melià y Sesé, s. f.) "han confirmado la contribución de los factores de naturaleza psicosocial y organizacional a la seguridad".

En la actualidad, se cuentan con estudios a nivel individual de las variables aplicación de la norma G050, seguridad durante la construcción, y clima de seguridad, entre los cuales podemos destacar: (1) "Mejoramiento de la Norma G050 para disminuir los accidentes durante la construcción Perú - 2018". Tacna, Perú, Universidad Privada de Tacna, («Duarte, P.», 2018), cuyo autor desarrollo un estudio con el fin de mejorar la norma G050 seguridad durante la construcción para disminuir los accidentes durante la construcción; (2) "Culture and climate of safety in organizations: conceptualizations and assessment". Saskatchevan, Canada, University of Saskatchewan, (Kaczur, M., 2017), cuyo autor estudió los conceptos de clima de seguridad y cultura de seguridad con el propósito de abordar los problemas conceptuales de tales constructos, (3) "Acciones encaminadas a mejorar la percepción de los factores psicosociales del clima de seguridad y salud en la Unión Eléctrica". La Habana, Cuba, Universidad de la Habana, (Viera, Y., s. f.), cuyo autor realizó una investigación respecto al clima de seguridad y salud del trabajo (SST) y su relación con los factores psicosociales que inciden en el mismo, obteniendo como resultados que la no implicación del personal, las dificultades en la comunicación y la motivación son las variables que más influyen en la percepción del clima de seguridad; asimismo, evidenció la necesidad de implantar un sistema de reconocimiento y estimulación del trabajo, a efectos de superar las dificultades en la comunicación eficaz y, el desinterés con el proceder ante la exigencia de la seguridad. Finalmente, el estudio concluye precisando que los resultados obtenidos ofrecen un apoyo empírico relacionado a la influencia de variables socio psicológicas en el estado del Clima de seguridad; (4) "Safety culture and safety climate definitions suitable for a regulator a sistematic literature review", Monash University and Institute for Safety Compensation and Recovery Research (2014) ( Vu, T. \& Cieri, H. De, 2014) cuyo autor realizó una revisión sistemática del concepto clima de seguridad, y presenta un resumen de los principales modelos de cultura y clima de seguridad; (5) "Medida del clima de seguridad y salud laboral", Publicaciones de la Universidad de Murcia, (Melià y Sesé, s. f.), cuyos autores precisan que el clima de seguridad es "la percepción del trabajador acerca del conjunto de acciones hacia la seguridad realizadas por la empresa, con efectos sobre la conducta de los trabajadores"; asimismo, diseñaron para su medición el cuestionario $\mathrm{C} 3 / 15$, el cual según sus autores considera las variables: “i) Estructuras de seguridad de la empresa, ii) Política de seguridad de la empresa, y iii) Acciones específicas en seguridad y salud laboral", a efectos de determinar elementos de intervención para la mejora del estado de seguridad de las organizaciones laborales y la prevención de enfermedades profesionales, accidentes laborales y sus consecuencias.

A pesar de lo señalado en el párrafo anterior, no se cuenta con estudios para determinar la influencia de la aplicación de las leyes y normas en materia de seguridad y salud en el trabajo, y en particular la norma G050, en el clima de seguridad en el sector construcción, siendo esto una limitante para la autoridad administrativa de trabajo en su rol regulador, y para las empresas del sector construcción en la adecuación de sus políticas a las leyes y normas de seguridad y salud en el trabajo del sector.

Estando a lo expuesto, el presente estudio tiene por objeto evaluar la influencia de la aplicación de la Norma G050 en el clima de seguridad de las obras de construcción del distrito de Surquillo, y de este modo contribuir en un sentido práctico al mejor control de la variable comportamiento hacia la seguridad y coadyuvar a la prevención de accidentes de trabajo en las obras de construcción en el distrito de Surquillo, más aun considerando el creciente desarrollo de este sector en el último año y los accidentes fatales registrados en los últimos años; asimismo, en un sentido teórico, el presente estudio, propone un modelo de medición de los factores que influyen en la aplicación de la norma G050 - Seguridad durante la construcción, el mismo que puede ser un punto de partida para que los investigadores que deseen comprender las razones del cumplimiento de las diversas normas de seguridad y salud en el trabajo (legales o propias de su organización), puedan adaptarla y aplicarla al ámbito de su investigación, lo que representa un aporte a la seguridad y salud en el trabajo

\section{MÉTODOS}

La unidad de análisis en el estudio la conforman los trabajadores que prestan servicios en veinticinco (25) obras 
multifamiliares de construcción del distrito de Surquillo; asimismo, se seleccionó una muestra aleatoria de ciento noventa y seis (196) trabajadores para la evaluación de las variables "Influencia de la aplicación de la Norma G050" y "Clima de Seguridad de las Obras de Construcción del Distrito de Surquillo"; cabe indicar que, estando a que las obras de construcción visitadas presentan características diversas, tales como el tipo de empresa (microempresa, pequeña empresa, y gran empresa), etapa de la construcción en la que se encuentra (demolición, excavación, construcción, acabados, entre otros), tipo de construcción (autoconstrucción, construcción a cargo de inmobiliarias, entre otros), no fue posible determinar de modo exacto la población, estando a ello el cálculo de la muestra se realizó a partir de una población desconocida.

Estando a lo señalado se empleó la fórmula de cálculo de muestra a partir de una población desconocida, la cual es:

$$
n=\frac{Z_{\propto}^{2} \cdot p \cdot q}{i^{2}}
$$

Cabe indicar además que, al no contar con marcos de muestreo previos, se utilizó un porcentaje estimado de $50 \%$, es decir se asume que "p" y "q" serán 50\%, y que resulta lo más común, cuando seleccionamos por primera vez una muestra en una población.

Donde:

n: tamaño muestral

Z: valor correspondiente a la distribución de gauss,

p: prevalencia esperada del parámetro a evaluar, para nuestro caso $\mathrm{p}=0.5$

$\mathrm{q}: 1-\mathrm{p}=0.5$

i: error que se prevé cometer si es del $7 \%, \mathrm{i}=0.7$

$\mathrm{n}=(1.96)^{2} \mathrm{x}(0.5) \times(0.5)$

$\mathrm{n}=196$

Estando a lo expuesto la muestra calculada es 196 trabajadores.

El presente trabajo de investigación se enmarca en el tipo de investigación aplicada, que tiene como propósito resolver un problema respondiendo a un diseño no experimental, caracterizado por la observación de los fenómenos en su ambiente natural para luego analizarlos. Finalmente, la investigación consideró al cuestionario como técnica de investigación, la misma que en función a las variables objeto de análisis presentó las siguientes características:

Cuestionario CEFIC-G050 de evaluación de factores, tiene por objeto evaluar si las variables legitimidad, normas personales/sociales, identidad social, y disuasión influyen en el cumplimiento de la norma G050, y en el clima de seguridad de las obras de construcción del distrito de Surquillo. Cabe indicar que, las citadas variables fueron tomadas a partir del estudio de (Frías Armenta,Martín Rodríguez y Corral Verdugo, s. f.), y adaptadas a modo que pudieran ser aplicables a los comportamientos que influyen en el cumplimiento de la norma G050. Cabe señalar que, los referidos autores definen a las variables legitimidad, normas personales/sociales, identidad social, y disuasión según se detalla a continuación: “i) legitimidad. - la cual se define como el cumplimiento voluntario de las normas a partir de la percepción de que la ley o las autoridades son justas; ii) norma personal y social. -se define norma personal a los estándares de la gente, internalizados a partir de las normas sociales, y cuya autocategorización resulta de la identificación al grupo social y sus normas, y se define norma social como los estándares morales sociales externos al individuo, pero que pueden internalizarse por este último transformándose en normas personales que condicionaran el comportamiento; iii) identidad social. -se define como el grado de identificación de los individuos a una familia, un grupo, o un lugar, hecho que influye en las normas sociales y personales, y la legitimidad; y iv) disuasión. - la disuasión se refiere a la amenaza de sanción o castigo con el propósito de hacer cumplir la ley; asimismo, la teoría de la disuasión señala que la decisión de realizar actividades ilegales está asociada a la probabilidad de ser detectado y sancionado por sus infracciones, y por la magnitud de estas últimas".

Cuestionario C3/15: Cuestionario de clima de seguridad: El cuestionario "Clima de Seguridad de las Obras de Construcción del Distrito de Surquillo", es una herramienta consistente en una serie preguntas sistematizadas en función a los componentes del Clima de Seguridad, y tiene por objeto reunir datos que permitan determinar el nivel de influencia de la variable "Influencia de la aplicación de la norma G050" hacia la variable "Clima de Seguridad de las Obras de Construcción del distrito de Surquillo". Cabe indicar que la citada encuesta fue tomada del estudio de (Melià y Sesé, s. f.) , quién estructuró el cuestionario en un modelo trifactorial que contiene las siguientes dimensiones: "i) Estructuras de seguridad (canales de comunicación, comités y representantes de seguridad y salud); ii) Política de seguridad (prioridad por la seguridad, rapidez vs. seguridad), y iii) Acciones de intervención en seguridad (carteles, cursillos o charlas, reuniones de trabajo, sistema de incentivos, instrucciones, e inspecciones en seguridad)".

\section{RESULTADOS}

\subsection{Análisis y resultados de la variable influencia de la aplicación de la norma G050}

Este estudio analizó los cuatro indicadores que confirman esta variable: Legitimidad de la norma G050 (Tabla 1), Norma personal/social respecto a la norma G050 (Tabla 2), Identidad social respecto a la norma G050 (Tabla 3), Disuasión respecto al cumplimiento de la norma G050 (Tabla 4) se consolidó con la apreciación general de la variable (Tabla 5). Se empleó el cuestionario CEFIC-G 050 como instrumento de análisis. 
La Tabla 1, muestra la distribución de la valoración legitimidad de la norma G 050 (Brioso, X., 2009), y en detalle evidencia que la percepción de legitimidad de autoridades tiene un valor mayor de $4.01 \mathrm{y}$, la percepción de la legitimidad del proceso tiene un valor de 3.93; asimismo, se tiene que el promedio general de la valoración legitimidad de la norma G050 tiene un valor promedio de 3.97 .

Tabla 1. Valoración de la legitimidad respecto a la norma G050

\begin{tabular}{cccc}
\hline $\mathbf{N}^{\circ}$ & Indicadores del Análisis & $\sigma$ & $\%$ \\
\hline 1 & Percepción de legitimidad de autoridades & 4.01 & $80.26 \%$ \\
2 & Percepción de legitimidad del proceso & 3.93 & $78.50 \%$ \\
& & 3.97 & $79.38 \%$ \\
\hline
\end{tabular}

Fuente: Elaboración propia

La Tabla 2, muestra la distribución de la valoración la norma personal/social respecto a la norma G050, y en detalle evidencia que reprobación social tiene un valor de 3.76 , la norma personal tiene un valor de 3.90 , la conducta antisegura tiene un valor de $2.52 \mathrm{y}$, la probabilidad de conducta antisegura tiene un valor de 2.68; asimismo, se tiene que el promedio general de la valoración de la norma personal/social respecto a la norma G050 es de 3.22.

Tabla 2. Valoración de la norma personal/social respecto a la norma G050

\begin{tabular}{clcc}
\hline $\mathbf{N}^{\circ}$ & \multicolumn{1}{c}{ Indicadores del Análisis } & $\sigma$ & $\%$ \\
\hline 1 & Reprobación social & 3.76 & $75.10 \%$ \\
2 & Norma personal & 3.90 & $78.08 \%$ \\
3 & Conducta antisegura & 2.52 & $50.42 \%$ \\
4 & Probabilidad de conducta antisegura & 2.68 & $53.62 \%$ \\
& & 3.22 & $64.30 \%$ \\
\hline
\end{tabular}

Fuente: Elaboración propia

La Tabla 3, muestra la distribución de la valoración de la identidad social respecto a la norma G050, y en detalle evidencia que la identidad social tiene un valor de 3.16; asimismo, se tiene que el promedio general de la valoración de la norma personal/social respecto a la norma G050 es de 3.16.

Tabla 3. Valoración de la identidad social respecto a la norma G050

\begin{tabular}{cccc}
\hline $\mathbf{N}^{\circ}$ & Indicadores del Análisis & $\sigma$ & $\%$ \\
\hline 1 & Identidad social & 3.16 & $63.24 \%$ \\
& & 3.16 & $63.24 \%$ \\
\hline
\end{tabular}

Fuente: Elaboración propia

La Tabla 4, muestra la distribución de la disuasión respecto a la norma G050, y en detalle evidencia que la probabilidad de sanción tiene un valor de 3.99, la severidad de la sanción tiene un valor de 3.88 , y la percepción de la ilegalidad de la conducta tiene un valor de 3.51; asimismo, se tiene que el promedio general de la valoración de la disuasión respecto a la norma G050 es de 3.79 .
Tabla 4. Valoración de la disuasión respecto a la norma G050

\begin{tabular}{clcc}
\hline $\mathbf{N}^{\circ}$ & \multicolumn{1}{c}{ Indicadores del Análisis } & $\overline{\mathbf{X}}$ & $\%$ \\
\hline 1 & Probabilidad de sanción & 3.99 & $79.73 \%$ \\
2 & Severidad de la sanción & 3.88 & $77.55 \%$ \\
3 & Percepción de ilegalidad de conducta & 3.51 & $70.14 \%$ \\
& & 3.79 & $75.80 \%$ \\
\hline
\end{tabular}

Fuente: Elaboración propia

La Tabla 5, muestra la distribución de la valoración general de la influencia de la aplicación de la norma G050 $\mathrm{y}$ en detalle evidencia que la legitimidad tiene un valor de 3.97 , la norma personal/social tiene un valor de 3.22, la identidad social tiene un valor de $3.16 \mathrm{y}$, la disuasión tiene un valor de 3.79 ; asimismo, se tiene que el promedio de la valoración general de la influencia de la aplicación de la norma G050 es de 3.53 .

Tabla 5. Valoración general de la influencia de la aplicación de la Norma G050

\begin{tabular}{clcc}
\hline $\mathbf{N}^{\circ}$ & \multicolumn{1}{c}{ Indicadores del Análisis } & $\sigma$ & $\%$ \\
\hline 1 & legitimidad & 3.97 & $79.33 \%$ \\
2 & norma personal/social & 3.22 & $64.30 \%$ \\
3 & identidad social & 3.16 & $63.24 \%$ \\
4 & disuasión & 3.79 & $75.80 \%$ \\
& & 3.53 & $70.67 \%$ \\
\hline
\end{tabular}

Fuente: Elaboración propia

\subsection{Análisis y resultados de la variable Clima de seguridad de las obras de construcción del distrito de Surquillo}

Este estudio analizó los tres indicadores que conforman esta variable: estructuras de la seguridad (Tabla 6), nivel de seguridad (Tabla 7) y, Acciones de intervención de seguridad (Tabla 8); se consolida con la apreciación general de la variable en la Tabla 9. Se empleó el cuestionario C3/15 como instrumento de análisis.

Tabla 6. Valoración de estructuras de seguridad

\begin{tabular}{clcc}
\hline $\mathbf{N}^{\circ}$ & \multicolumn{1}{c}{ Indicadores del Análisis } & $\sigma$ & $\%$ \\
\hline 1 & $\begin{array}{l}\text { Conozco procedimientos para informar a la dirección } \\
\text { de las condiciones inseguras en mi área de trabajo. }\end{array}$ & 3.35 & $66.94 \%$ \\
\hline $\begin{array}{l}\text { En mi empresa existen comités de seguridad e } \\
\text { higiene del trabajo. }\end{array}$ & 4.63 & $92.55 \%$ \\
$3 \quad \begin{array}{l}\text { En mi empresa existe un departamento o un } \\
\text { encargado de seguridad e higiene en el trabajo. }\end{array}$ & 3.33 & $66.53 \%$ \\
\hline $\begin{array}{l}\text { Conozco a los representantes de los comités de } \\
\text { seguridad e higiene en el trabajo de mi empresa. }\end{array}$ & 3.39 & $67.76 \%$ \\
\hline $\begin{array}{l}\text { Conozco las funciones de los comités de seguridad e } \\
\text { higiene en el trabajo. }\end{array}$ & 3.33 & $66.63 \%$ \\
\hline
\end{tabular}

Fuente: Elaboración propia

La Tabla 6, muestra la distribución de las estructuras de seguridad, y en detalle evidencia que el punto 1: "Conozco procedimientos para informar a la dirección de las condiciones inseguras en mi área de trabajo" tiene un 
valor de 3.35; el punto 2: "En mi empresa existen comités de seguridad e higiene del trabajo" tiene un valor de 4.63; el punto 3: "En mi empresa existe un departamento o un encargado de seguridad e higiene en el trabajo" tiene un valor de 3.33; el punto 4: "Conozco a los representantes de los comités de seguridad e higiene en el trabajo de mi empresa" tiene un valor de 3.39 ; y el punto 5 : "Conozco las funciones de los comités de seguridad e higiene en el trabajo" tiene un valor de 3.33; asimismo, se tiene que el promedio general de la valoración de las estructuras de seguridad es de 3.60 .

La Tabla 7, muestra la distribución de la Valoración de las Políticas de seguridad, y en detalle evidencia que el punto 1: "Los aspectos de seguridad son una prioridad dentro de los objetivos de la empresa" tiene un valor de 3.29; el punto 2: "En el desempeño de mi trabajo esta primero la seguridad que la rapidez." tiene un valor de 3.61; el punto 3: "Se me han dado a conocer las políticas sobre seguridad e higiene de mi empresa" tiene un valor de 3.48 y; el punto 4: "Mi empresa muestra mucho interés en la seguridad" tiene un valor de 3.31; asimismo, se tiene que el promedio general de la valoración de las políticas de seguridad es de 3.42 .

Tabla 7. Valoración de las Políticas de Seguridad

\begin{tabular}{clcc}
\hline $\mathbf{N}^{\circ}$ & \multicolumn{1}{c}{ Indicadores del Análisis } & $\sigma$ & $\%$ \\
\hline 1 & $\begin{array}{l}\text { Los aspectos de seguridad son una prioridad dentro } \\
\text { de los objetivos de la empresa. }\end{array}$ & 3.29 & $65.71 \%$ \\
2 & $\begin{array}{l}\text { En el desempeño de mi trabajo esta primero la } \\
\text { seguridad que la rapidez. }\end{array}$ & 3.61 & $72.14 \%$ \\
& $\begin{array}{l}\text { Se me han dado a conocer las políticas sobre } \\
\text { seguridad e higiene de mi empresa. }\end{array}$ & 3.48 & $69.69 \%$ \\
\hline $\mathbf{N}^{\circ}$ & Indicadores del Análisis & 0 & $\%$ \\
\hline 4 & Mi empresa muestra mucho interés en la seguridad. & 3.31 & $66.12 \%$ \\
\hline
\end{tabular}

Fuente: Elaboración propia

La Tabla 8, muestra la distribución de las estructuras de seguridad, y en detalle evidencia que el punto 1: "En mi empresa hay carteles sobre seguridad en el trabajo" tiene un valor de 3.48; el punto 2: "Mi empresa realiza cursillos o charlas sobre seguridad" tiene un valor de 3.55; el punto 3: "Hacemos reuniones de trabajo específicas sobre problemas de seguridad" tiene un valor de 3.34; el punto 4: "Mi empresa tiene un sistema para recompensar, incentivar o premiar el trabajar de forma segura" tiene un valor de 3.42; el punto 5: "Recibimos indicaciones e instrucciones orales o escritas sobre seguridad" tiene un valor de $3.25 \mathrm{y}$; el punto 6: "En mi empresa se realizan inspecciones para el control de las condiciones de seguridad" tiene un valor de 3.37; asimismo, se tiene que el promedio general de la valoración de las acciones de intervención de seguridad es de 3.40 .

La Tabla 9, muestra la distribución general del Clima de seguridad de las obras de construcción del distrito de Surquillo, y en detalle evidencia que el punto 1: "Estructuras de la Seguridad" tiene un valor de 3.60; el punto 2: "Políticas de seguridad" tiene un valor de 3.42 y; el punto 3: "Acciones de intervención de seguridad" tiene un valor de 3.40; asimismo, se tiene que el promedio general de la valoración general del clima de seguridad es de 3.48 .

Tabla 8. Valoración de acciones de intervención de seguridad

\begin{tabular}{clcc}
\hline $\mathbf{N}^{\circ}$ & \multicolumn{1}{c}{ Indicadores del Análisis } & $\sigma$ & $\%$ \\
\hline 1 & $\begin{array}{l}\text { En mi empresa hay carteles sobre seguridad en el } \\
\text { trabajo. }\end{array}$ & 3.48 & $69.69 \%$ \\
2 & $\begin{array}{l}\text { Mi empresa realiza cursillos o charlas sobre } \\
\text { seguridad. }\end{array}$ & 3.55 & $70.92 \%$ \\
3 & $\begin{array}{l}\text { Hacemos reuniones de trabajo específicas sobre } \\
\text { problemas de seguridad. }\end{array}$ & 3.34 & $66.73 \%$ \\
4 & $\begin{array}{l}\text { Mi empresa tiene un sistema para recompensar, } \\
\text { incentivar o premiar el trabajar de forma segura. }\end{array}$ & 3.42 & $68.47 \%$ \\
5 & $\begin{array}{l}\text { Recibimos indicaciones e instrucciones orales o } \\
\text { escritas sobre seguridad. }\end{array}$ & 3.25 & $65.00 \%$ \\
6 & $\begin{array}{l}\text { En mi empresa se realizan inspecciones para el } \\
\text { control de las condiciones de seguridad. }\end{array}$ & 3.37 & $67.45 \%$ \\
\hline
\end{tabular}

Fuente: Elaboración propia

Tabla 9. Valoración general del clima de seguridad de las obras de construcción del distrito de Surquillo

\begin{tabular}{clcc}
\hline $\mathbf{N}^{\circ}$ & \multicolumn{1}{c}{ Indicadores del Análisis } & $\sigma$ & $\%$ \\
\hline 1 & Estructuras de la Seguridad & 3.6 & $72.08 \%$ \\
2 & Políticas de seguridad & 3.42 & $68.42 \%$ \\
3 & Acciones de intervención de seguridad & 3.40 & $68.04 \%$ \\
& & 3.48 & $69.51 \%$ \\
\hline
\end{tabular}

Fuente: Elaboración propia

\subsection{Relación general entre las variables Aplicación de la norma G050 y clima de seguridad de las obras de construcción del distrito de Surquillo}

A efectos de determinar la relación entre las variables V1: Aplicación de la norma G050 y V2: clima de seguridad de las obras de construcción del distrito de Surquillo (Tablas 5 y 9) se empleó la prueba del chi cuadrado $\mathrm{X}^{2}$, con el propósito de evaluar los efectos y/o relación existente; para tal fin se realizaron los siguientes pasos:

Paso 1: Formulación de las hipótesis nula y alternativa:

Hipótesis Estadísticas:

- $\mathrm{h}_{1}$. La aplicación de la norma G050 influye significativamente en el Clima de Seguridad de las Obras de Construcción del Distrito de Surquillo

- $\quad \mathrm{h}_{0}$ : La aplicación de la norma G050 NO influye significativamente en el Clima de Seguridad de las Obras de Construcción del Distrito de Surquillo.

Paso 2: Selección del nivel de significancia:

Para nuestro caso el nivel de significancia es 0.01 . La probabilidad que se rechace la hipótesis nula verdadera es 0.01 .

Paso 3: Selección del estadístico chí cuadrado (Tabla 10): 
Para nuestro caso se empleó la prueba del chi cuadrado $\mathrm{X}^{2}$, cuya fórmula es:

$$
X^{2}=\frac{\sum(o-e)^{2}}{e}
$$

Donde:

$\mathrm{o}=$ Frecuencia observada

$\mathrm{e}=$ Frecuencia esperada

Paso 4: Formulación de la regla de decisión:

\section{Región de Rechazo:}

La $\mathbf{h}_{0}$ será rechazada si el valor observado (calculado) de $\mathrm{X}^{2}$ es tal que la probabilidad asociada con su realización, para un grado de libertad: $\mathrm{gl}=6,(\mathrm{~N}-1)$, es igual o menor para el valor tabulado de $\mathrm{X}^{2}$ para el nivel de significación $\alpha=0.01$.

A partir de los siguientes datos se calcula $\mathrm{x}^{2}$ tabulada:

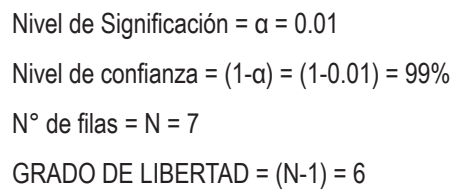

Tabla 10. Tabla para valores chi cuadrado crítico (extracto)

\begin{tabular}{cccc}
\hline $\begin{array}{c}\text { grados de } \\
\text { libertad }\end{array}$ & $\mathbf{0 . 0 0 5}$ & $\mathbf{0 . 0 1}$ & $\mathbf{0 . 0 2 5}$ \\
\hline 1 & 0.00003935 & 0.000157 & 0.000982 \\
2 & 0.01 & 0.02 & 0.051 \\
3 & 0.072 & 0.115 & 0.216 \\
4 & 0.207 & 0.297 & 0.484 \\
5 & 0.412 & 0.554 & 0.831 \\
6 & 0.676 & 0.872 & 1.237 \\
7 & 0.989 & 1.239 & 1.69 \\
8 & 1.344 & 1.647 & 2.18 \\
\hline
\end{tabular}

Fuente: Elaboración propia

Estando a los cálculos realizados se tiene que $\mathrm{x}^{2}$ tabulada es 0.872 .

Por tanto, la regla de decisión sería: $\quad 0$

$\underline{X^{2}} \underline{2}$ calculado $>X^{2} \underline{2}$ tabulado; entonces se rechaza Ho (Hipótesis Nula) y por tanto se comprueba hipótesis de investigación

$\underline{X^{2}} \underline{\text { calculado }<X^{2}} \underline{\text { tabulado }}$; entonces se acepta Ho (Hipótesis Nula)

Paso 5: Cálculo del chi cuadrado:

Para realizar el cálculo del chi cuadrado se preparó una tabla con las siguientes columnas, y se sistematizo en una base de datos excel su fórmula de cálculo:
Tabla 11. Relación general entre las variables Aplicación de la norma G050 y clima de seguridad de las obras de construcción del distrito de Surquillo

\begin{tabular}{cccccc}
\hline 1.0 & $(\mathrm{Oi})$ & $(\mathrm{Ei})$ & $\underline{\mathrm{Oi}-\mathrm{Ei}}$ & $\underline{\underline{(\mathrm{Oi}-\mathrm{Ei})^{2}}}$ & $\frac{(\mathrm{Oi}-\mathrm{Ei})^{2}}{\underline{\underline{\mathrm{Ei}}}}$ \\
\hline 1 & 3.97 & 5.00 & -1.03 & 1.07 & 0.21 \\
2 & 3.22 & 5.00 & -1.78 & 3.19 & 0.64 \\
3 & 3.16 & 5.00 & -1.84 & 3.38 & 0.68 \\
4 & 3.79 & 5.00 & -1.21 & 1.46 & 0.29 \\
1 & 3.60 & 5.00 & -1.40 & 1.95 & 0.39 \\
2 & 3.42 & 5.00 & -1.58 & 2.49 & 0.50 \\
3 & 3.40 & 5.00 & -1.60 & 2.55 & 0.51 \\
& 24.56 & 35.00 & & & 3.22 \\
\hline
\end{tabular}

Fuente: Elaboración propia

Donde:

Oi: frecuencias observadas

Ei: frecuencias esperadas

$$
X^{2}=\frac{\sum(o-e)^{2}}{e}=3.34
$$

Estando a los cálculos realizados se tiene que $\mathrm{X}^{2}$ calculado es 3.22.

\section{PASO 6: Toma de decisión}

Finalmente, toda vez que, $\mathrm{X}^{2}$ calculada es mayor que $\mathrm{X}^{2}$ tabulada, se rechaza la hipótesis nula $\mathbf{h}_{0}$, y se acepta la $\mathrm{h}_{1}$. Estando a ello se tiene que: "La aplicación de la norma G050 influye significativamente en el Clima de Seguridad de las Obras de Construcción del Distrito de Surquillo”.

\section{DISCUSIÓN}

\subsection{Influencia de la aplicación de la norma G050 en el Clima de Seguridad de las Obras de Construcción del Distrito de Surquillo}

Según el objetivo general y de acuerdo a los resultados obtenidos en el presente estudio, podemos indicar que la aplicación de la norma G050 influye en el Clima de Seguridad de las Obras de Construcción del Distrito de Surquillo; por cuanto, a partir de la prueba de hipótesis se demuestra una relación de dependencia de la variable V2 (Clima de seguridad de las obras de construcción del distrito de Surquillo) respecto a variable V1 (Aplicación de la norma G050).

Estos resultados guardan relación con la tesis presentada por («Gyves, M.», 2014), cuyo estudio consistió en "identificar los factores que determinan la gestión de la seguridad y salud ocupacional en la empresa, para posteriormente analizar su impacto en el clima de seguridad de los trabajadores de las plantas de producción". La referida autora, identifica como uno de los factores para la gestión de la seguridad y salud ocupacional a las "acciones de las dependencias gubernamentales y su contribución al cumplimiento de la normatividad de la 
empresa", evidenciando además en sus resultados que "la gestión de la seguridad y salud se encuentra determinada por el cumplimiento de la normatividad"; finalmente, entre sus principales conclusiones señala que "la evaluación favorable del clima de seguridad en una de sus plantas se explica por su alto nivel de cumplimiento normativo", evidenciándose por tanto coincidencias significativas con el presente estudio.

Con estos resultados, podemos afirmar que ambos estudios demuestran que existe una influencia entre el cumplimiento normativo y el clima de seguridad, lo que podría determinar un mejor control de las conductas hacia la seguridad, de parte de la gestión de seguridad y salud en el trabajo, y de este modo contribuir a la prevención de accidentes de trabajo en las obras de construcción en el distrito de Surquillo, más aun considerando el creciente desarrollo de este sector en los últimos años y los accidentes fatales registrados en los años 2014 y 2018.

\subsection{La aplicación de la norma G050 está relacionada a condiciones que influyen en su cumplimiento}

El promedio general de la valoración legitimidad de la norma G050 es de 3.97, de la norma personal/social respecto de la norma G050 es de 3.22, de la identidad social respecto de la norma G050 es de 3.16, y de la disuasión respecto de la norma G050 es de 3.79; superando en todos los casos el valor central esperado de 3; por tanto, dichas condiciones representan un valor positivo; $y$ refieren que los entrevistados señalan que: i) consideran legitimo el cumplimiento de la citada norma, ello basado en la percepción de que la norma G050 y las autoridades responsables de su verificación son justas, ii) el cumplimiento de la citada norma está asociada a sus normas personales/sociales; es decir, los trabajadores señalan que cumplen la norma G050 por estar alineada a sus normas personales/sociales, iii) el cumplimiento de la citada norma se basa en su autocategorización como miembros de un grupo social, hecho que además influye en las normas sociales y personales, y en la legitimidad de la norma G050; iv) el cumplimiento de la citada norma se basa en la probabilidad de que la conducta infractora sea detectada, y la certeza de recibir una sanción por dicho incumplimiento.

El promedio general de la valoración de la influencia de la aplicación de la Norma G050 es de 3.53, superando el valor central esperado de 3, por tanto, tal valoración representa un valor positivo; y refieren que los entrevistados señalan que cumplen la citada norma por la percepción de legitimidad de la norma G050 y las autoridades encargadas de su fiscalización, las normas personales y sociales que regulan su cumplimiento, la identidad social o autocategorización a un grupo social, y la disuasión ante la amenaza de sanción por su incumplimiento; por tanto, se tiene que la aplicación de la norma G050 está asociada a la valoración general de la legitimidad, norma personal/social, identidad social y disuasión, respecto a la norma G050 de parte de los trabajadores de las obras de construcción del distrito de Surquillo.

Estos resultados guardan relación con («Pajuelo, K.», 2011)quiénseñalaque"lafaltadesanciónporincumplimiento de las normas de seguridad origina reincidencias en dichas indisciplinas", asimismo, pone en evidencia la legitimidad de los trabajadores hacia las autoridades a través de la siguiente expresión contenida en su investigación: "Si el jefe ve que él hace mal, él tiene que sancionar para que no lo vuelva a hacer, sino el trabajador dice no me dicen nada, entonces lo sigo haciendo y hago lo que yo quiero". Por otro lado, (CONSSAT, s. f.) en el informe final del estudio denominado "Percepciones y actitudes hacia la salud y seguridad en el trabajo de construcción civil", señala que: 1) el compañerismo es una herramienta útil para reforzar la conducta segura, 2) la conducta segura del trabajador está vinculada al posible llamado de atención, suspensión o separación, y por tanto en el temor de desproteger a la familia, y 3) la familia es un tema importante para los trabajadores y es un tema que se toca constantemente en las capacitaciones diarias; por lo expuesto, podemos afirmar que los estudios señalados demuestran que la legitimidad hacia las normas y autoridades, las normas personales y sociales, la identidad social, y la disuasión ante la amenaza de sanción por su incumplimiento son elementos que contribuyen al cumplimiento de las normas de seguridad en el sector construcción, y en particular de la norma G050, evidenciándose por tanto coincidencias significativas con el presente estudio.

\subsection{El clima de seguridad en las obras de construcción del distrito de Surquillo está relacionado a condiciones que influyen en su comportamiento}

El promedio general de las estructuras de seguridad, políticas de seguridad, y acciones de intervención de seguridad presentan una calificación de $3.60,3.42$, y 3.40 respectivamente, superando el valor central esperado de 3 , por tanto, dichas condiciones representan un valor positivo; sin perjuicio de ello, se evidencia que hay aspectos que reforzar respecto a las citadas condiciones; comparativamente, el estudio de (Viera, Y., s. f.) presenta un promedio general de las estructuras de seguridad, políticas de seguridad, y acciones de intervención de seguridad presentan una calificación de 4.08, 4.07, y 3.94, coincidiendo con nuestra investigación toda vez que, se supera el valor central esperado de 3, demuestra un valor positivo, y detecta oportunidades de mejora respecto a este factor. Cabe añadir, que los valores obtenidos también coinciden con el estudio (CONSSAT, s. f.) toda vez que, si bien es cierto los trabajadores tienen conocimiento de la existencia de diversas estructuras de seguridad, políticas de seguridad, y acciones de intervención, es también cierto que refieren que hay aspectos que mejorar en su funcionamiento.

\section{CONCLUSIONES}

- La aplicación de la norma G050 influye en el Clima de Seguridad de las Obras de Construcción del Distrito de Surquillo, tal como se demuestra en la prueba de hipótesis del presente estudio.

- El promedio general de la valoración de la influencia de la aplicación de la Norma G050 es de 3.50, superando el valor central esperado de 3, representando por tanto un valor positivo; estando a lo expuesto, se tiene que la aplicación de la norma G050 está asociada a la valoración general de la 
legitimidad, norma personal/social, identidad social y disuasión que perciben los trabajadores respecto a la norma G050 en las obras de construcción del distrito de Surquillo.

- El promedio general de la valoración del Clima de seguridad es de 3.48, superando el valor central esperado de 3, representando por tanto un valor positivo; estando a lo expuesto, se tiene que el comportamiento del clima de seguridad está asociado a la valoración general de las estructuras de la Seguridad, de las políticas de seguridad, y de las acciones de intervención de seguridad de parte de los trabajadores de las obras de construcción del distrito de Surquillo.

- Los valores de las condiciones legitimidad, la norma personal/social, la identidad social, y la disuasión respecto a la norma G050 son 3.97, 3.22, 3.16 , y 3.79 respectivamente; superando en todos los casos el valor central esperado de 3; por tanto, dichas condiciones representan un valor positivo, y refieren que la aplicación de la norma G050 está asociada a la valoración general de las citadas condiciones de parte de los trabajadores de las obras de construcción del distrito de Surquillo.

- Los valores de las condiciones de estructuras de seguridad, políticas de seguridad, y acciones de intervención de seguridad del clima de seguridad son $3.60,3.42$, y 3.40 respectivamente; superando en todos los casos el valor central esperado de 3 ; por tanto, dichas condiciones representan un valor positivo, y refieren que el clima de seguridad está asociada a la valoración general de las citadas condiciones de parte de los trabajadores de las obras de construcción del distrito de Surquillo.

\section{AGRADECIMIENTOS}

Al Ph.D Carlos Cabrera Carranza por sus consejos y ánimos para continuar con la presente investigación, a mi asesor Mg. Oskar Huapaya Ramírez y a mi revisor metodológico Mg. José López Kohler por su paciencia y valiosa guía a pesar de las restricciones de tiempo, a mi profesor de pregrado Mg. Edgar Vicente Armas por su soporte en la parte estadística de la investigación, a todo el personal docente y administrativo de la Facultad de Ingeniería Geológica, Minera, Metalúrgica y Geográfica de la UNMSM por todo su apoyo brindado.

\section{REFERENCIAS}

América Noticias. (s. f.). América Noticias. Recuperado 7 de junio de 2020, de https://www.americatv.com.pe/noticias/ actualidad/dos-obreros-murieron-electrocutados-rozarvarilla-cable-alta-tension-n152235
Bird, F. y Germain, G. (s. f.). Recuperado 7 de junio de 2020, de https://www.academia.edu/33509861/Liderazgo_ Pr\%C3\%A1ctico_en_el_Control_de_P\%C3\%A9rdidas

Brioso, X. (2009, noviembre 1). https://www.researchgate. net/publication/316853137_APLICACION_Y COMPATIBILIZACION DE $\bar{L}$ A NORMA G050 SEGURIDAD_DURANTE_LA_CONSTRUCCION

CONSSAT. (s. f.). Informe Final Recuperado 7 de junio de 2020, de https://www.trabajo.gob.pe/archivos/file/ CNSST/ESTUDIO_CUALITATIVO_PERCEPCIONES ACTITUDES_SST_CONSTRUCION_CIVIL.pdf

Duarte, P. (2018). Repositorio Institucional - UPT. http://renati. sunedu.gob.pe/handle/sunedu/286335

El Comercio Perú. (2018, septiembre 30) https://elcomercio.pe/ lima/policiales/surquillo-obrero-muere-caer-septimo-pisoobra-construccion-noticia-nndc-563073-noticia/

Frías Armenta,Martín Rodríguez y Corral Verdugo. (s. f.). Recuperado 7 de junio de 2020, de https://www.redalyc.org/ pdf/284/28412891012.pdf

Gyves, M. (s. f.). Tesis para obtener el grado de Maestria en Administracion Integral del Ambiente, México 2014, pp.124. https://www.colef.mx/posgrado/wp-content/ uploads/2014/11/TESIS-Pati\%C3\%B1o-De-GyvesMariana.pdf

Kaczur, M. (2017). [Thesis, University of Saskatchewan]. https:// harvest.usask.ca/handle/10388/8187

Meliá y Sesé. (s. f.). Recuperado 7 de junio de 2020, de https:// www.redalyc.org/pdf/167/16715211.pdf

MTPE. Boletin Estadístico Mensual -Edición Agosto 2019Notificaciones de accidentes de trabajo, incidentes peligrosos y enfermedades ocupacionales. (s. f.). Recuperado 7 de junio de 2020, de https://www.gob.pe/institucion/mtpe/informespublicaciones/307055-boletin-estadistico-mensual-edicionagosto-2019-notificaciones-de-accidentes-de-trabajoincidentes-peligrosos-y-enfermedades-ocupacionales

Municipalidad de Surquillo. (s. f.). Recuperado 7 de junio de 2020, de http://www.munisurquillo.gob.pe/licencia_edificacion/ lehu_pdf/LICENCIA_DE_EDIFICACION_2016.pdf

Pajuelo, K. (2011). Pontificia Universidad Católica del Perú. http:// tesis.pucp.edu.pe/repositorio/handle/20.500.12404/934

Viera, Y. (s. f.). studylib.es. Recuperado 7 de junio de 2020, de https://studylib.es/doc/5200600/título--accionesencaminadas-a-mejorar-la-percepción-de-los

Vu, T. \& Cieri, H. De. (2014). Safety culture and safety climate definitions suitable for a regulator. A systematic literature review pp. 92. https://research.iscrr.com.au/_data/assets/ pdf_file/0005/292316/Literature-review-safety-culture-andclimate-definitions-for-regulators.pdf 